\title{
Care of patients with stomas in general practice
}

Ben Finlay, Hannah Sexton, Christopher McDonald

\section{Background}

Intestinal stomas are formed for emergency, elective, benign and malignant conditions. They may be temporary or permanent. The complication rates of intestinal stomas are reported as high as $56 \%$.

\section{Objective}

The aim of this article is to provide an overview of intestinal stomas and common related issues, to inform general practitioners (GPs) and improve stoma-related care.

\section{Discussion}

There are a variety of early and late complications associated with intestinal stomas. It is important that patients have access to an informed GP, stomal therapy nurse and surgeon to provide optimal ongoing care. Good stoma care contributes to good quality of life for patients.
A STOMA OR OSTOMY is an opening created between a hollow viscus and the skin. Common intestinal stomas encountered in primary care are ileostomies and colostomies. The creation of a stoma is a life-changing event for a patient and is usually borne out of a period of significant stress related to malignancy or illness. Aside from the physical change, a stoma may affect other aspects of wellbeing, including psychological and sexual health. In the primary care setting, an understanding of stomas and their complications is important in providing appropriate care for these patients.

The literature suggests that many doctors have a poor understanding of stomas and potential associated problems. ${ }^{1}$ Here, we discuss ileostomies and colostomies and relevant issues for primary care providers, including when to refer. Well-informed clinicians can assist patients in maintaining a good quality of life with their stoma and prevent avoidable complications.

Stomas may be temporary or permanent, depending on their indication and patient comorbidities. ${ }^{1,2}$ Indications for ileostomy and colostomy include bowel resection for benign, malignant or inflammatory bowel disease, congenital anomalies or continence problems. Potential complications are discussed below. It is important that clinicians have a basic understanding of stomas, are aware of the significance of certain examination findings and know when to refer to a surgeon or stomal therapy nurse (STN).

\section{Types of stomas}

An ileostomy is usually sited on the right side of the abdomen. Given the irritant nature of small bowel effluent, ideally, an ileostomy will pout $2-3 \mathrm{~cm}$ above the level of the skin into the stoma appliance ('bag'). A colostomy is usually sited on the left side of the abdomen and does not necessarily pout like an ileostomy.

Stomas can be either 'end', meaning only one lumen is brought to the skin, or 'loop', meaning both proximal and distal limbs are brought to the skin surface (Figure 1). Loop stomas are often created when reversal is planned in the future. Loop ileostomies are often formed for diversion after resection for rectal cancer or inflammatory bowel disease.

\section{Professionals involved in patient care}

Several health professionals are involved in caring for patients with stomas (Figure 2).

Surgical involvement is usually limited to early pre-operative and postoperative care. Ongoing management by a surgeon is often not required for the stoma specifically, unless there is a significant complication or the stoma is to be reversed.

As the primary care provider, the general practitioner (GP) provides comprehensive and continuing care and has a vital role in care coordination, support and referring patients with complications if necessary (Box 1).

An STN is a specialist in stoma, continence and wound management. ${ }^{3}$ They form an integral part of the team caring for a person with a stoma and are often the initial contact for patients experiencing problems. ${ }^{1,3}$ STNs can be contacted at most major hospitals, district nursing agencies and in private colorectal surgical practices.

Ideally, a patient will be seen by an STN pre-operatively to be counselled and sited for their stoma. ${ }^{1,4}$ When siting a stoma, the patient's abdominal contours and skin folds should be 
considered so that the stoma is visible to the patient and the appliance adheres well to skin. The bowel should pass through rectus abdominus to prevent herniation. ${ }^{2,5}$ In emergency situations it is not always possible to involve an STN pre-operatively.

Postoperatively, STNs are vital in providing patient education, selecting appropriate appliances, troubleshooting stoma problems and providing postoperative support, all of which optimise physical and psychological wellbeing.

\section{Complications of stomas}

Complications of stomas are relatively common, with the literature citing rates as high as $37-56 \% .^{1,6}$ Many complications are dependent on surgical factors, including site selection and stoma construction. ${ }^{1,5}$ However, diabetes, obesity and smoking may also predispose complications. ${ }^{7}$

Early complications include stomal necrosis, dehiscence and retraction..$^{1,2,5}$ Later complications include stomal stenosis, parastomal hernia and prolapse. Other complications that may occur at any time are bleeding, peristomal skin excoriation, bowel obstruction and high stomal output.

\section{Necrosis} stoma can represent an emergency. ${ }^{2,5}$ One needs to ascertain whether the blood supply to the stoma has been compromised, and if this represents wider mesenteric ischaemia or superficial mucosal
Usually an early complication, a dusky

ischaemia. Usually these patients should be referred to hospital for surgical review.

\section{Dehiscence}

Separation of the mucocutaneous junction is most common in the early postoperative period. ${ }^{5}$ This may require revision of the stoma and, given the risk of peristomal infection and faecal peritonitis, the patient should be sent for surgical review.

\section{Bleeding}

Blood in the stoma bag is a somewhat common presentation and may be alarming for the patients and clinicians. It is important to remove the bag and thoroughly examine the patient, including a digital stoma examination, to ascertain whether the source is superficial or gastrointestinal.

Superficial bleeding granulomas at the mucocutaneous junction may be treated with the application of silver nitrate. Check whether the appliance itself is causing trauma. ${ }^{8}$ Peristomal bleeding may be due to varices, which can usually be stopped with cautery or suture. ${ }^{2}$ Blood from within the stoma should be treated as for any gastrointestinal bleeding.

\section{Retraction and stenosis}

Over time, the patient's stoma may become sunken, or the skin opening may narrow. ${ }^{2}$ One must maintain suspicion for an inflammatory or malignant cause. ${ }^{2}$

Stomal retraction, defined as $\geq 0.5 \mathrm{~cm}$ below skin level, may occur as an early or late complication. ${ }^{9}$ Retraction early in the postoperative period is usually caused by excessive tension on the bowel related to poor site selection, inadequate surgical
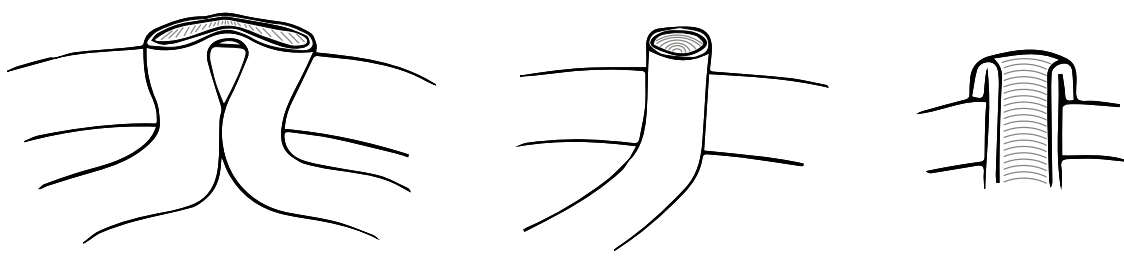

Figure 1. Schematic diagram illustrating loop stoma (left), end stoma (centre) and matured end stoma (right) mobilisation or obesity. Late retraction is usually a result of chronic ischaemia, also usually in the context of obesity. It is best to refer these patients to an STN or surgeon for review.

\section{Prolapse}

A patient may present with a length of gut suddenly appearing within their stoma bag. This should be reduced as soon as possible to prevent compromise of the prolapsed segment. It is reasonable to attempt to reduce the prolapse in the primary care setting if the prolapse appears healthy. Applying table sugar to reduce oedema may help with reduction. However, if this is unsuccessful or the gut appears compromised, the patient should be sent to hospital for surgical review. While distressing for the patient and clinician, it is rarely a surgical emergency.

\section{Parastomal hernia}

A parastomal hernia occurs when there is protrusion of abdominal contents through the parastomal fascial defect. Patients will notice a bulge around their stoma. This is more likely to occur in patients who are overweight or have a history of other hernias. ${ }^{10}$ It is a risk factor for peristomal dermatitis and ulceration due to difficulty in fitting appliances. Clinical evidence of bowel obstruction or incarceration requires urgent referral to hospital for surgical review.

\section{Box 1. Stoma-related complications requiring referral}

Urgent surgical review:

- Dusky stoma or stomal necrosis

- Stomal dehiscence

- Peristomal bleeding

- Bowel obstruction

- Incarcerated parastomal hernia

- New high-output stoma with associated dehydration

Timely review by stomal therapy nurse or surgeon:

- Stomal retraction or stenosis

- Parastomal hernia (without evidence of obstruction)

- Peristomal skin irritation

- Poorly fitting stoma appliances 


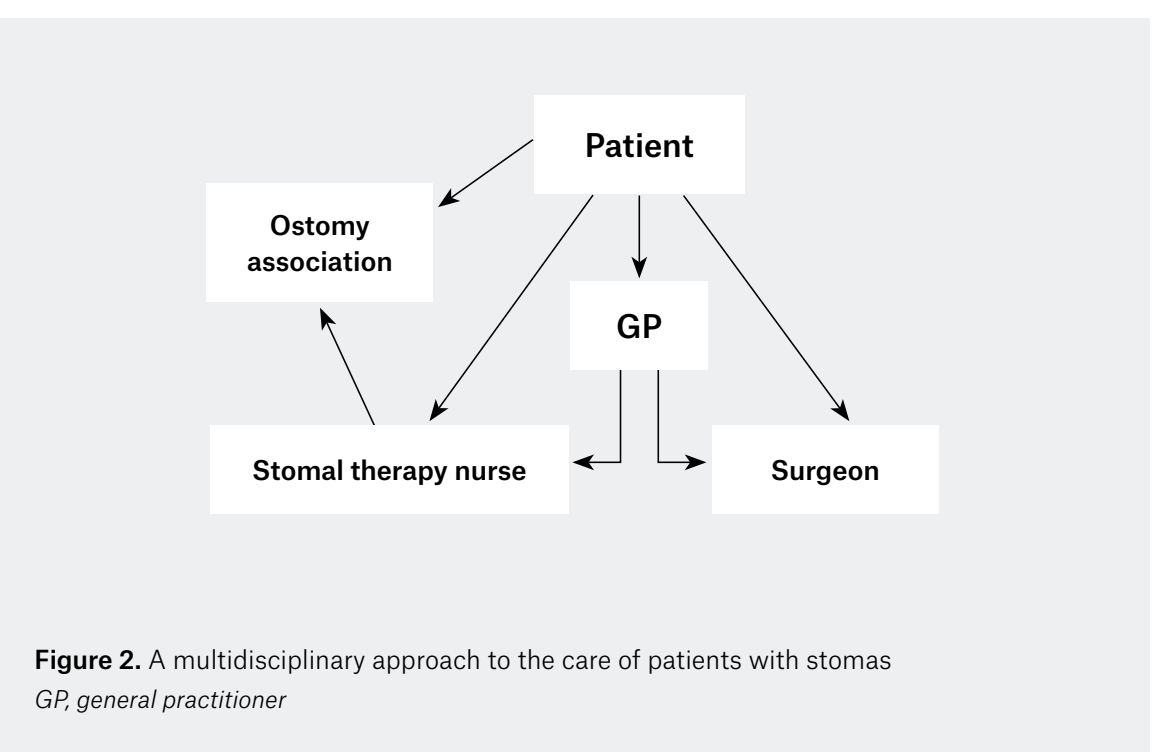

\section{Decreased stomal output}

Patients present after noting that they have not needed to empty their stoma bag as often as usual. It is important to take a thorough history (Box 2) and examination (Box 3) to differentiate between bowel obstruction and constipation. Examine for hernias that may be causing an obstruction. A digital stoma examination may reveal hard faeces obstructing the stoma, which a small enema may help relieve. If there is suspicion of bowel obstruction, the patient should be referred to hospital for surgical review.

\section{High-output stoma}

A high-output stoma is traditionally defined as stomal output of $>2000 \mathrm{~mL}$ per day. ${ }^{11}$ It is generally accepted that outputs $<1000 \mathrm{~mL}$ per day are desirable and $300-600 \mathrm{~mL}$ is safe. Patients with proximal ileostomies are particularly susceptible to dehydration and electrolyte imbalance due to loss of colonic water absorption. ${ }^{2}$ It is important that patients are cognisant of this and maintain adequate oral fluid intake. Most high-output stomas should reduce their output over time. ${ }^{11}$ Drugs such as loperamide and fibre supplements can be used to reduce stomal output, but a careful titration of these is required to avoid constipation. If patients become acutely dehydrated, they may require intravenous fluid therapy.

\section{Peristomal skin irritation}

Peristomal skin irritation and excoriation are common problems for people with stomas. ${ }^{2,5}$ STNs are experts at managing these conditions. A common cause of skin irritation is stomal effluent, usually due to a poorly fitting stoma bag. ${ }^{2,5}$ Consultation with an STN should be sought to optimise fitting. Another cause may be reaction to the stoma bag itself or the adhesive used. Frequent removal and reapplication of the appliance can also damage skin. ${ }^{5}$ An STN can identify hypoallergenic products or protective barriers to reduce such a reaction.

\section{Life impact of stomas}

The creation of a stoma often has a significant physical and psychological impact on a person. ${ }^{6,12,13}$ Perioperative counselling as a preventive measure is essential, but preventing complications is also important. ${ }^{3}$ Throughout Australia there are 22 regional ostomy associations dedicated to providing information, support and supplies for people with stomas (see http://australianstoma.com.au).

Odour is a common concern for people with stomas, but bags are designed to be odour-proof and inconspicuous. Odour may be minimised further by emptying the bag more frequently and changing the bag once or twice per week. Similarly, audible flatus may be minimised by dietary advice from an STN.

Most forms of physical activity, including swimming, and activities of daily living are safe when wearing a stoma pouch. Sexual activity is also safe and the psychological impact of the bag may be minimised with the use of a supportive brace. Extreme contact sports should be avoided.

\section{Conclusion}

As the primary care provider, it is important for the GP to have an understanding of intestinal stomas and their potential complications, and to know when to refer for surgical or stomal therapy advice. While a stoma may have a significant impact on a patient's life, with

\section{Box 2. History from a patient with a stoma}

How long has the patient had a stoma?

Why did they have a stoma created?

How do they manage with their stoma?

What impact does their stoma have on their daily life?

When was the last time they saw their surgeon or stomal therapy nurse?

How many times per day do they empty their stoma bag? Has that changed recently?

Are there plans from their surgeon for stoma reversal?

\section{Box 3. Useful points to note on examination of patient with a stoma}

Shape of abdomen

Location of stoma on abdomen

Type of appliances used

Contents of stoma bag

Appearance of stoma (ensure patient has another appliance to fit after the examination)

Is it pink and healthy?

Is it pouting beyond skin? Is it sunken?

Appearance of skin surrounding stoma

Peristomal tenderness

Digital examination of stoma 
the support of a multidisciplinary team, including STNs and the patient's surgeon, most patients are able to maintain normal work and social functioning and an excellent quality of life.

\section{Authors}

Ben Finlay BSc (BiomedSci), MD, Surgical Registrar, Royal Adelaide Hospital, Adelaide, SA. benpfinlay@ gmail.com

Hannah Sexton MBBS, CWH, FRACGP, General Practitioner, Adelaide, SA

Christopher McDonald, MBBS, FRACS. Colorectal and General Surgeon, Lyell McEwin Hospital,

Elizabeth Vale, SA

Competing interests: None.

Provenance and peer review: Not commissioned externally peer reviewed.

\section{References}

1. Hendren S, Hammond K, Glasgow SC, et al. Clinical practice guidelines for ostomy surgery. Dis Colon Rectum 2015;58(4):375-87. doi: 10.1097/ DCR.0000000000000347.
2. Shellito PC. Complications of abdominal stoma surgery. Dis Colon Rectum 1998;41(12):1562-72.

3. McGrath A. Stoma-associated problems: The important role of the specialist nurse. Br J Nurs 2017;26(5):S30-31. doi: 10.12968/ bjon.2017.26.5.S30.

4. Salvadalena G, Hendren S, McKenna L, et al. WOCN Society and AUA position statement on preoperative stoma site marking for patients undergoing urostomy surgery. J Wound Ostomy Continence Nurs 2015;42(3):253-56. doi: 10.1097/ WON.0000000000000118.

5. Kann BR. Early stomal complications. Clin Colon Rectal Surg 2008;21(1):23-30. doi: 10.1055/s2008-1055318.

6. Nastro P, Knowles CH, McGrath A, Heyman B, Porrett TR, Lunniss PJ. Complications of intestinal stomas. Br J Surg 2010;97(12):1885-89. doi: 10.1002/bjs.7259.

7. Persson E, Berndtsson I, Carlsson E, Hallén AM, Lindholm E. Stoma-related complications and stoma size - A 2-year follow up. Colorecta Dis 2010;12(10):971-76. doi: 10.1111/j.14631318.2009.01941.x.

8. Khan M, Schiff R. Parastomal hernia and stoma granuloma. BMJ (online) 2013;346(may22 18):f3178. doi: 10.1136/bmj.f3178.
9. Arumugam PJ, Bevan L, Macdonald L, et al. A prospective audit of stomas - Analysis of risk factors and complications and their management. Colorectal Dis 2003;5(1):49-52.

10. Krogsgaard M, Thomsen T, Vinther A, Gögenur I, Kaldan G, Danielsen AK. Living with a parastomal bulge - Patients' experiences of symptoms. J Clin Nurs 2017;26(23-24):5072-81. doi: 10.1111/ jocn.14009.

11. Baker ML, Williams RN, Nightingale JM. Causes and management of a high-output stoma. Colorectal Dis 2011;13(2):191-97. doi: 10.1111/j.14631318.2009.02107.x.

12. Williams J. Coping: Teenagers undergoing stoma formation. Br J Nurs 2017;26(17):S6-11. doi: 10.12968/bjon.2017.26.17.S6

13. Brown H, Randle J. Living with a stoma: A review of the literature. J Clin Nurs 2005;14(1):74-81.

correspondence ajgp@racgp.org.au 